\title{
Mechanisms of action of BCL6 during germinal center $B$ cell development
}

\author{
HUANG ChuanXin ${ }^{1 *} \&$ MELNICK Ari ${ }^{2 *}$ \\ ${ }^{1}$ Shanghai Institute of Immunology \& Department of Immunobiology and Microbiology, Shanghai Jiao Tong University School of Medicine, \\ Shanghai 200025, China; \\ ${ }^{2}$ Division of Hematology and Medical Oncology, Department of Medicine, Weill Cornell Medical College, New York NY 10065, USA
}

Received July 26, 2015; accepted August 17, 2015; published online November 13, 2015

\begin{abstract}
The transcriptional repressor B cell lymphoma 6 (BCL6) controls a large transcriptional network that is required for the formation and maintenance of germinal centers (GC). GC B cells represent the normal counterpart of most human B-cell lymphomas, which are often characterized by deregulated BCL6 expression or BCL6-mediated pathways. BCL6 suppresses gene transcription largely through recruitment of its co-repressors through its distinct repression domain. Understanding the precise biological roles of each repression domain in normal and malignant B cells is helpful for development of targeted inhibition of BCL6 functions that is emerging as the basis for design of anti-lymphoma therapies. This review focuses on recent progress in the molecular mechanisms of action of BCL6 in B cells and discusses remaining unresolved questions related to how these mechanisms are linked to normal and malignant B cell development.
\end{abstract}

BCL6, germinal center, co-repressor, lymphoma

Citation: Huang CX, Melnick A. Mechanisms of action of BCL6 during germinal center B cell development. Sci China Life Sci, 2015, 58: 1226-1232, doi: $10.1007 / \mathrm{s} 11427-015-4919-\mathrm{z}$

The germinal center $(\mathrm{GC})$ reaction, involving generation of high-affinity memory $\mathrm{B}$ cells and plasma cells, is the basis of T-dependent humoral immunity against foreign pathogens [1]. The GC reaction is highly dynamic and coordinated by multiple immune cell types including $\mathrm{T}$ cells, B cells and follicular dendritic cells [2]. GCs are transient microstructures formed within the follicles of secondary lymphoid tissues during the GC reaction. GC B cell development is highly dynamic and instructed by specific and intricate transcriptional programs [3]. Notably, some of the core molecular mechanisms driving these programs are also involved in B-cell malignant transformation. Indeed, the majority of human mature $\mathrm{B}$ cell lymphomas originate from GC B-cells [4].

The transcriptional repressor B cell lymphoma 6 (BCL6),

*Corresponding author (email: huangex@shsmu.edu.cn; amm2014@med.cornell.edu) originally identified as encoded by a frequently translocated locus in diffuse large B cell lymphomas (DLBCLs) [5], serves as a master regulator of the GC reaction [6,7]. BCL6 is essential for the development and function of both GC B cell and $\mathrm{T}$ follicular helper (Tfh) cell [8-10], a specialized T helper subtype which supports B cells during the GC reaction. Loss of BCL6 in each cell type results in abrogation of the GC reaction. BCL6 acts as an oncogene in GC-derived B-cell lymphoma, which is usually characterized by deregulated BCL6 expression due to chromosomal translocation or mutations of its promoter, or feature genetic lesions in pathways normally regulated by BCL6 [11,12].

From the biochemical mechanism standpoint, BCL6 exerts its effects mostly through recruitment of various co-repressor complexes [13]. These different co-repressor complexes appear to suppress distinct gene sets, perhaps mediating different activities of BCL6 in various immune 
cells. This notion has been in part supported by studies using inhibitors that selectively disrupt the co-repressors binding to the BCL6 BTB (BR-C, ttk and bab) domain [14,15]. However, the link between the transcriptional mechanisms of action of BCL6 with its biological actions in the immune system remains to be further characterized. In recent years, two new mouse models designed to dissect the biochemical mechanisms of action of BCL6 have provided critical insight into the ways that BCL6 can control the phenotype of $\mathrm{B}$ cells during the $\mathrm{GC}$ reaction $[16,17]$.

\section{GC B cell development and B cell lym- phomagenesis}

Formation of GCs is central to the establishment of long-lived, high-affinity $\mathrm{T}$ cell-dependent $\mathrm{B}$ cell responses [1]. The first step towards GC formation is activation of naïve B cells by exogenous T-cell dependent antigen in the follicles of peripheral lymphoid tissues. Next, antigen-engaged B-cells migrate to the border between the follicles and the $\mathrm{T}$ cell zone or interfollicular zones, where they undergo proliferation and interact with cognate Tfh cells within 1-3 days after stimulation [18-21]. B-cells can then differentiate into extrafollicular plasma cells and leave the follicle to generate low-affinity antibodies, or alternatively can enter the GC pathway [22]. These early GC precursor B cells (i.e. pre-GC $\mathrm{B}$ cells) begin to up-regulate expression of BCL6 protein and move toward the center of lymphoid follicles [20,21]. BCL6 up-regulation in pre-GC B cells contributes to their sustained interactions with Tfh cells and is required for their further differentiation in the lymphoid follicles. Pre-GC B cells finally aggregate into early GC clusters at day 4 post-immunization.

Once early GCs are established, B cells undergo rapid proliferation, resulting in massive expansion of GCs. By seven days the GC becomes fully formed and is polarized between two microenvironments called the dark zone and the light zone. Dark zone B cells (centroblasts) undergo clonal expansion and somatic hypermutation (SHM) of immunoglobulin variable region to generate mutant clones with a broad range of affinities for the antigen, and then migrate towards the light zone $[2,22,23]$. Within the light zone GC B-cells (centrocytes) undergo three main differentiation processes: the positive selection of $\mathrm{B}$ cells expressing high-affinity immunoglobulin by Tfh cells, the process of class-switch recombination (CSR) to produce various types of immunoglobulin and the initiation of terminal differentiation into memory cells or plasma cells. Some centrocytes may re-enter the dark zone to undergo further rounds of proliferation, affinity maturation and selection.

Single and double strand DNA damage mediated by activation induced cytosine deaminase (AICDA) is required for SHM and CSR during the GC reaction and essential to permit immunoglobulin mutagenesis and maturation. How- ever, AICDA can also cause genetic alterations at genes beyond the immunoglobulin loci, resulting in the deregulated or ectopic expression of oncogenes or the inactivation of tumor suppressors. Accordingly, the majority of mature B cell lymphomas, including follicular lymphomas (FLs), DLBCLs and Burkett's lymphomas (BLs), arise from GC B cells [4], as shown by the presence of somatically mutated immunoglobulin genes in their genomes. Importantly, many of the somatic mutations occurring in these lymphomas are associated with genes that exhibit strong differential regulation during GC B cell development, such as MYC, BCL6 and PRDMI(which encodes B lymphocyte-induced maturation protein 1) [24]. Exploration of the functions of these genes during normal GC B cell development may thus be likely to provide a better understanding of the pathogenesis of B-cell lymphomas.

\section{BCL6: a master transcriptional regulator of GC B-cell development}

GC B cell differentiation is tightly controlled by various transcription regulators including BCL6 [3,24]. In the B-cell lineage, BCL6 protein is induced and highly expressed in centroblasts, and its expression is maintained in most centrocytes [25]. Bcl6-deficient mice fail to form GCs and hence are unable to generate high-affinity antibodies, demonstrating an essential requirement of this transcriptional factor in the establishment and/or maintenance of GCs $[6,7,26]$. One of the best characterized biological functions of BCL6 in centroblasts is to facilitate simultaneous rapid proliferation and tolerance of genomic damage occurring during clonal expansion and somatic hypermutation. BCL6 mediates these effects by directly repressing DNA damage sensing and checkpoint genes such as DNA damage sensor ATR (ataxia telangiectasia and Rad3 related), TP53 (tumor protein p53) tumor suppressor and cell cycle arrest gene CDKN1A (cyclin-dependent kinase inhibitor 1A) [27-29]. In addition, BCL6 blocks premature activation by $\mathrm{T}$ cells or other soluble signals through inhibiting a number of pathways involved in B-cell activation in T-cell dependent immune [11-13]. This function of BCL6 may be important to prevent centroblasts to exit from the GC before they complete the phase of proliferative expansion and of antibody affinity maturation. Finally, BCL6 represses a number of genes required for the differentiation of B cells into plasma cells, including PRDM1, a transcription factor that is crucial for plasma cell development [30,31].

Although the importance of BCL6 in determining the GC phenotype has been well defined, the functions of this protein in regulating the fate of pre-GC B cell have only recently begun to be understood. Recent studies using Bcl6-reporter mice and high-resolution intravital cellular imaging have established a critical role for BCL6 in pre-GC 
events [20,21]. BCL6 protein is first detected in the interfollicular zone in a small subset of activated antigen-engaged $B$ cells that have been successfully engaged by Tfh cells. Many of these BCL6-positive cells (i.e. pre-GC B cells) are destined to the GC pathway. BCL6 up-regulation appears to be important for pre-GC B cell to form sustained interaction with cognate Tfh cells and move towards the center of the follicle. BCL6 seems to drive pre-GC B cell migration at least in part through directly inhibiting the trafficking factors Sphingosine 1-phosphate receptor 1 (S1PR1) and GPR183, which encodes $G$ protein-coupled receptor 183 [21,31]. These new findings point to multifaceted biological functions of BCL6 in the GC response.

BCL6 must be switched off for GC B cell to exit from the GC and undergo terminal differentiation [32]. Deregulated BCL6 expression, due to chromosomal translocation or mutations of its own promoter, or genetic alterations in pathways normally regulated by BCL6, is commonly associated with DLBCLs and less frequently associated with FLs $[11,33,34]$. The pro-survival and proliferation functions of BCL6 appear to make GC B cells prone to malignant transformation. Mice constitutively expressing BCL6 in GC B cells develop DLBCL with features similar to the human tumors [35]. Indeed, most GC-derived lymphomas are dependent on the expression and transcriptional activity of BCL6 [14,31]. Thus, targeted inhibition of BCL6 functions has been proposed as a promising therapeutic strategy for GC-derived lymphomas.

\section{Biochemical mechanisms of action of BCL6}

The transcriptional repression activity of BCL6 is dependent on its binding to co-repressor proteins, and there are many co-repressors that BCL6 can partner with. BCL6 recruits co-repressors mainly via its conserved $\mathrm{N}$-terminal BTB/POZ (Pox virus and Zinc finger) domain and a middle unstructured region (often called the second repression domain or "RD2" domain) [13]. Both the BTB and RD2 domain recruit distinct sets of co-repressors (Figure 1). The BCL6 BTB domain forms an obligate homodimer to create two identical and symmetrical extended lateral grooves that form docking sites for the co-repressor proteins SMRT (silencing mediator of retinoid and thyroid receptor), NCOR(nuclear receptor corepressor) and BCOR (BCL6 corepressor) (Figure 1) [36,37]. NCOR and SMRT are highly conserved scaffold/adaptor proteins, both of which can individually form a stable complex with HDAC3 (histone deacetylase 3), TBL1 (transducin-beta-like protein 1), TBLR1 (transducin-beta-like related protein 1) and GPS2 (G protein pathway suppressor 2) [38]. Both NCOR and SMRT bind to BCL6 via a conserved 17-residue BCL6 binding domain (BBD) [37]. BCOR forms a completely different type of complex than NCOR and SMRT [39].

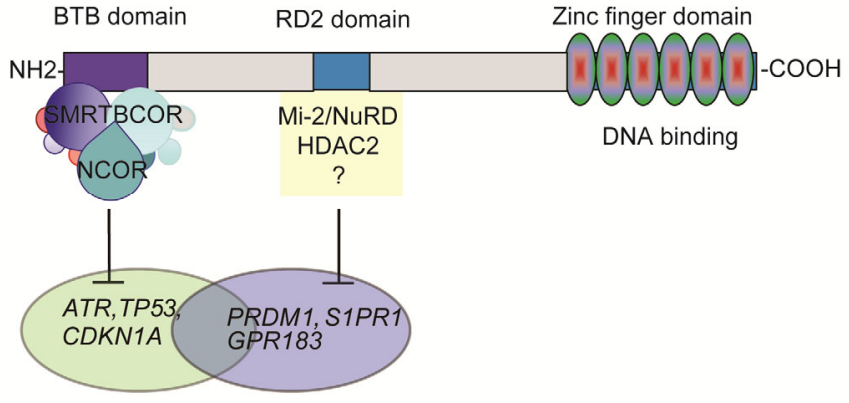

Figure 1 Schematic representation of key transcriptional mechanisms of action of BCL6. BCL6 exerts its repression activity largely through the recruitment of co-repressors mainly via $\mathrm{N}$-terminal BTB domain and second middle RD2 domain. The BTB domain co-repressors include SMRT, NCOR and BCOR, whereas the RD2 domain binds to HDAC2 and MTA3/NurD complex, perhaps other unknown con-repressors. BCL6 recruit distinct co-repressor complex to different subsets of targets.

Specifically, BCOR associates with a polycomb/ubiquitin ligase complex containing RING1, RYBP, NSPC1, RNF2, FBXL10 and Skp1 (S-phase kinase-assocated protein 1) [40]. The BBDs of NCOR and SMRT are identical, whereas the BCOR BBD is completely different, yet all three bind to the BCL6 BTB lateral groove in perfectly overlapping configurations [37,39]. BCL6 appears to recruit two distinct repression complexes at promoters and enhancers via its BTB domain [41]. At promoters, BCL6 dimers can recruit a unique ternary BCOR-SMRT/NCOR complex to effectively repress transcription in a repressed chromatin environment. At enhancers, BCL6 selectively recruits HDAC3-containing SMRT/NCOR complex to functionally inactivate these elements through H3K27 deacetylation. The RD2 domain recruits HDAC2 (histone deacetylase 2) and MTA3/NuRD (nucleosome remodeling and deacetylase) complex, and possibly other co- repressors (Figure 1) [30,42]. Interestingly, the co-activator p300 can bind and acetylate the RD2 domain at its KKYK motif and inhibit its transcription activity [42]. Recently, a 45-amino acid region compassing KKYK motif was identified to interact with HDAC2 and MTA3/NurD complex [17]. This region displayed a similar repression effect as full-length RD2. Finally, BCL6 recruits the co-repressor CtBP1 (C-terminal binding protein 1) via both the BTB and RD2 domain [43].

The transcriptional repression activity of BCL6 requires its binding to specific DNA elements. Recently, wholegenome chromatin immunoprecipitation plus sequencing (ChIP-seq) analysis identified genome-wide BCL6 binding sites $[33,41,44]$. BCL6 binding sites were shown to be significantly enriched in the BCL6 consensus binding motif TTCCT(A/C)GAA that was initially identified in vitro by examining the ability of recombinant BCL6 protein to bind to synthetic oligonucleotides. Hence BCL6 is often directly recruited to these regions through its $\mathrm{C}$-terminal zinc fingers. BCL6 can also bind to DNA indirectly through interacting with other BTB-containing transcription factors. For 
instance, BCL6 binding to the promoter of two genes (CDKN1A and BCL2) is dependent on ZBTB17 (also known as Miz1) [29,44]. BCL6 is recruited to the promoter of PRDM1 through BACH2 (BTB and CNC homologue 2) [45].

Meanwhile, ChIP-seq analysis also revealed that the BCL6 activities are associated with other transcription factors that are important regulators of GC B cell development. This is evident by this fact that $\mathrm{BCl} 6$ binding sites are significantly coincident with the cistrome of these proteins including MYB ( $\mathrm{v}$-myb myeloblastosis viral oncogene homology), IRF8 (interferon-regulatory factor 8) and BACH2 $[41,44,45]$. BCL6 seems to antagonize or synergize these transcription factors in programing the GC phenotype. For instance, BCL6 cooperates with BACH2 to suppress PRDM1 transcription and terminal differentiation [45]. Finally, BCL6 may suppress transcription by competing for binding with STAT family transcriptional activators, which share quite similar binding sites with BCL6 [6,16,46]. In summary, BCL6 serves as a transcriptional repressor through DNA binding and recruitment of co-repressor complexes.

\section{The BCL6 BTB domain is critical for prolif- eration and survival of mature GC B-cells}

Although the BCL6 target genes are well defined [33,41,44], the precise functions of separate BCL6 repression domain is less clear. BCL6 appears to recruit distinct co-repressor complex to different subsets of targets (Figure 1) [47,48], suggesting that transcriptional programming by BCL6 may be finely compartmentalized through distinct domains. A SMRT-based cell penetrating BCL6 peptide inhibitor (BPI), which is designed to dissociate the BCL6 BTB domain and its co-repressors [14], was able to induce expression of genes such as ATR, TP53 and CDKN1A involved in DNA damage and cell cycle checkpoints in primary GC B cells and DLBCL cells, but did not affect expression of BCL6 target genes involved in differentiation such as PRDMI [48]. Consistent with this, BPI administration prevented GC formation in mice and induced cell cycle arrest and apoptosis in DLBCL cells [14,47]. These studies suggest that the BTB domain is especially required for cell survival and proliferation.

A genetically engineered strain of mice was generated to express a mutant form of BCL6 that is unable to bind to SMRT, NCOR and BCOR, but is otherwise functionally intact, further supporting this notion [16]. These mice only formed very small GCs in response to T-cell dependent antigen stimulation due to a severe defect in GC B cell proliferation and survival. Further ChIP-seq studies in primary human GC B-cells demonstrated that BCL6 binds to key checkpoint genes ATR, TP53, and CDKN1A together with SMRT and/or BCOR [16]. Hence, the role of BCL6 as a regulator of cell survival and proliferation is principally mediated through the BTB domain and is mostly relevant to its physiological actions in GC B-cells (Figure 2). The same mechanism is maintained during tumorigenesis, as demonstrated by the fact that GC-derived lymphomas are sensitive to the BCL6 BTB inhibitors [14,15,49]. Unexpectedly, BTB loss of function did not result in significant impairment of Tfh cell development and function as well as aberrant differentiation of other $\mathrm{T}$ helper subtypes [16]. All tested inflammatory cytokines were expressed at comparable levels

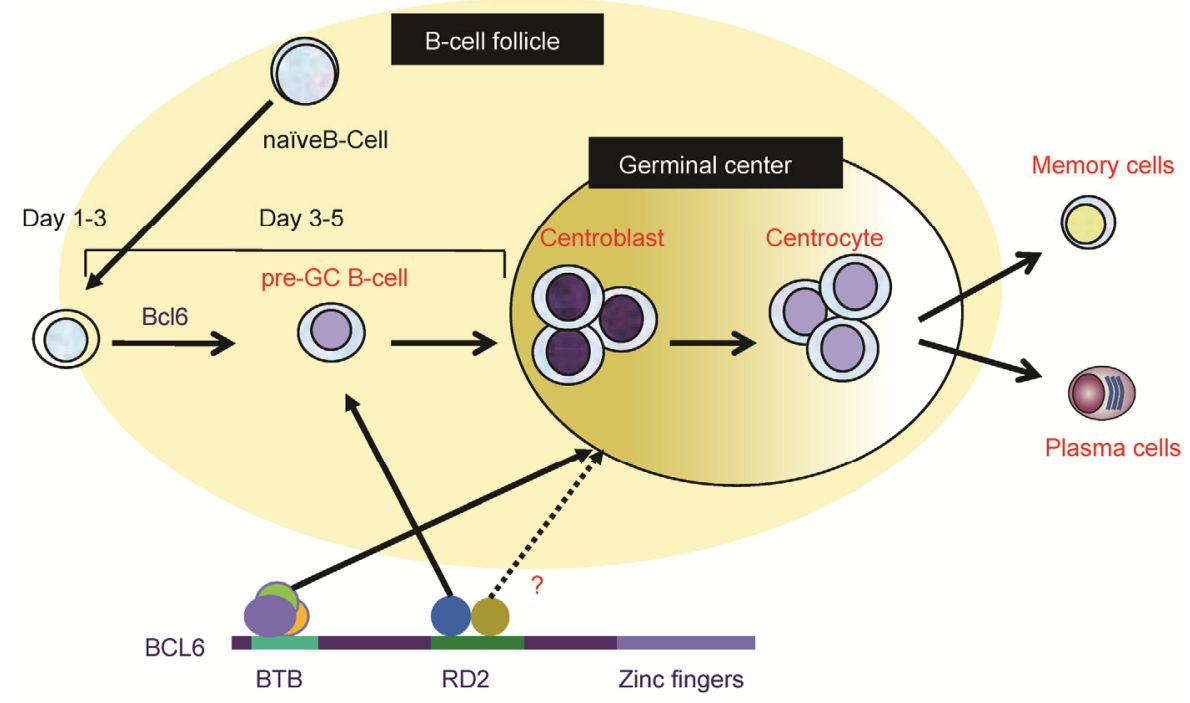

Figure 2 The proposed biochemical mechanisms of action of BCL6 during GC B cell development. The development of GC B cells is a sequential and complex process, and tightly controlled by BCL6. The BTB domain is critical for survival and proliferation of mature GC B-cells, whereas the RD2 domain plays an essential role in the development and clustering of early GC-committed B-cells. The role of the RD2 domain is mature GC B cells remains to be further investigated. 
by macrophages derived from BTB mutant mice. Accordingly, these animals lived normal, healthy lives and displayed no evidence of the lethal inflammatory disease that is characteristic of BCL6 deficient mice $[6,16]$. Therefore, the molecular functions of BCL6 in T cells and macrophages are less dependent on the BTB domain repression function. BCL6 competing for binding with STAT family protein may present a dominant biochemical function of BCL6 in restricting inflammatory gene expression [16]. Other unknown repression mechanisms, perhaps through the RD2 domain, may compensate for BTB loss of function to maintain the phenotype of $\mathrm{T}$ cells.

\section{The BCL6 RD2 domain is essential for pre-GC B-cell differentiation}

Recent studies have defined an essential role of BCL6 in pre-GC events, especially for sustained T-B interaction and pre-GC B cell migration [21]. However, how BCL6 acts on these pre-GC B-cells remains unknown. BTB-mutant B cells can still form early GCs [16], indicating that BTB repression function is dispensable for pre-GC B-cell development (Figure 2). However, mice engineered to express a Bcl6 mutant that lost RD2 domain repression function exhibit a complete loss of GC formation but retain normal extrafollicular responses [17]. High-resolution intravital cellular imaging showed that RD2-mutant antigen-engaged B-cells can normally migrate to the interfollicular zone and interact with cognate $\mathrm{T}$ helper cells after immunization. However, these cells failed to complete early GC-commitment differentiation and coalesced as nascent GC aggregates [17]. The profound defect in early pre-GC differentiation in RD2-deficent B cells best explains the complete abrogation of GC formation in Bcl6-deficent mice and emphasizes the importance of Bcl6 in early GC B-cell commitment (Figure 2). RD2 controls migration at least in part through directly suppressing expression of the key migration factor GRP183 [17], down-regulation of which is critically important for B-cell migration into the follicle center. In addition, BCL6 directly represses SIPRI and indirectly induces $S 1 P R 2$ expression. S1PR1 enables B cells to move out of the follicles, whereas S1PR2 confines B cells to an S1P-low niche in the center of follicles [50]. Through dynamic regulation and equilibrium of S1PR1 and S1PR2, the BCL6 RD2 domain seems to enable "capture" of B-cells within follicles to promote their clustering into an S1P-low region.

From the mechanistic standpoint, the BCL6 RD2 domain suppresses the transcription of the GPRI83 and SIPRI genes by recruiting HDAC2, but not MTA3/NuRD to the enhancer active mark $\mathrm{H} 3 \mathrm{~K} 27 \mathrm{ac}$ at their distal regulatory elements [17]. Collectively, the BCL6 RD2 domain mediates early steps in commitment to the GC fate and clustering into nascent GCs, whereas the BCL6 BTB domain is required to maintain the proliferation and survival by repressing genes during the later GC stage (Figure 2). These findings suggest a model of sequential and biochemically distinct biological functions of BCL6 at different GC B-cell developmental stages.

\section{Conclusions and perspectives}

Recent studies on the mechanisms of action of BCL6 are clarifying important questions regarding the stages and biochemical mechanisms through which BCL6 controls GC B-cell development. It is becoming clear that the BTB and $\mathrm{RD} 2$ repression domains have distinct effects on the phenotype of B cells during the GC reaction (Figure 2). Interestingly, the BTB and RD2 domains have limited effects on the phenotype of $\mathrm{T}$ cells and macrophage. These studies point towards striking biochemical specificity in BCL6 control of humoral and innate immune-cell phenotypes and provide a new paradigm for transcription factor functional diversification.

Although the BCL6 RD2 domain is critical in regulating the fate of pre-GC B cells, its precise roles in B cells within established GCs remain to be answered (Figure 2). The BCL6 RD2 was reported to recruit MTA3-NurD complex to suppress $P R D M 1$ transcription to prevent terminal differentiation of $\mathrm{B}$ cells into plasma cells in cultured lymphoma cells, suggesting that the RD2 domain might be important to prevent terminal differentiation of B-cells in established GC. The RD2 co-repressor MTA3 was found to be dispensable to inhibit genes implicated in cell cycle and apoptosis; however we cannot exclude the possibility that other unknown RD2 co-repressors modulate proliferation and survival of GC B-cells as well as lymphoma. The new mice model in which RD2 loss of function is specifically introduced in mature GC B-cells are urgent to assess these questions. Elucidation of new functions of RD2 domain in B cell lymphomas may lead to the identification of novel therapeutic targets for these diseases. Finally, it is possible that both BTB- and RD2-associated co-repressors bind to some BCL6 target sites and failure to recruit one or the other of each domain-specific co-repressor do not sufficiently impair its repression function. The biological importance of this biochemical mechanism needs to be further examined. Perhaps this is required for BCL6 to prevent premature activation.

The author(s) declare that they have no conflict of interest.

1 MacLennan IC. Germinal centers. Annu Rev Immunol, 1994, 12: 117-139

2 Victora GD, Nussenzweig MC. Germinal centers. Annu Rev Immunol, 2012, 30: 429-457

3 De Silva NS, Klein U. Dynamics of B cells in germinal centres. Nat 
Rev Immunol, 2015, 15: 137-148

4 Küppers R. Mechanisms of B-cell lymphoma pathogenesis. Nat Rev Cancer, 2005, 5: 251-262

5 Ye BH, Lista F, Lo Coco F, Knowles DM, Offit K, Chaganti RS, Dalla-Favera R. Alterations of a zinc finger-encoding gene, BCL-6, in diffuse large-cell lymphoma. Science, 1993, 262: 747-750

6 Dent AL, Shaffer AL, Yu X, Allman D, Staudt LM. Control of inflammation, cytokine expression, and germinal center formation by BCL-6. Science, 1997, 276: 589-592

7 Ye BH, Cattoretti G, Shen Q, Zhang J, Hawe N, de Waard R, Leung C, Nouri-Shirazi M, Orazi A, Chaganti RS, Rothman P, Stall AM, Pandolfi PP, Dalla-Favera R. The BCL-6 proto-oncogene controls germinal-centre formation and Th2-type inflammation. Nat Genet, 1997, 16: 161-170

8 Nurieva RI, Chung Y, Martinez GJ, Yang XO, Tanaka S, Matskevitch TD, Wang YH, Dong C. Bcl6 mediates the development of T follicular helper cells. Science, 2009, 325: 1001-1005

9 Yu D, Rao S, Tsai LM, Lee SK, He Y, Sutcliffe EL, Srivastava M, Linterman M, Zheng L, Simpson N, Ellyard JI, Parish IA, Ma CS, Li QJ, Parish CR, Mackay CR, Vinuesa CG. The transcriptional repressor BCL-6 directs $\mathrm{T}$ follicular helper cell lineage commitment. Immunity, 2009, 31: 457-468

10 Johnston RJ, Poholek AC, DiToro D, Yusuf I, Eto D, Barnett B, Dent AL, Craft J, Crotty S. Bcl6 and Blimp-1 are reciprocal and antagonistic regulators of $\mathrm{T}$ follicular helper cell differentiation. Science, 2009, 325: $1006-1010$

11 Basso K, Dalla-Favera R. Roles of BCL6 in normal and transformed germinal center B cells. Immunol Rev, 2012, 247: 172-183

12 Hatzi K, Melnick A. Breaking bad in the germinal center: how deregulation of BCL6 contributes to lymphomagenesis. Trends Mol Med, 2014, 20: 343-352

13 Ci W, Polo JM, Melnick A. B-cell lymphoma 6 and the molecular pathogenesis of diffuse large B-cell lymphoma. Curr Opin Hematol, 2008, 15: 381-390

14 Polo JM, Dell'Oso T, Ranuncolo SM, Cerchietti L, Beck D, Da Silva GF, Prive GG, Licht JD, Melnick A. Specific peptide interference reveals BCL6 transcriptional and oncogenic mechanisms in B-cell lymphoma cells. Nat Med, 2004, 10: 1329-1335

15 Cerchietti LC, Ghetu AF, Zhu X, Da Silva GF, Zhong S, Matthews $\mathrm{M}$, Bunting KL, Polo JM, Farès C, Arrowsmith CH, Yang SN, Garcia M, Coop A, Mackerell AD Jr, Privé GG, Melnick A. A smallmolecule inhibitor of BCL6 kills DLBCL cells in vitro and in vivo. Cancer Cell, 2010, 17: 400-411

16 Huang C, Hatzi K, Melnick A. Lineage-specific functions of Bcl-6 in immunity and inflammation are mediated by distinct biochemical mechanisms. Nat Immunol, 2013, 14: 380-388

17 Huang C, Gonzalez DG, Cote CM, Jiang Y, Hatzi K, Teater M, Dai K, Hla T, Haberman AM, Melnick A. The BCL6 RD2 domain governs commitment of activated B cells to form germinal centers. Cell Rep, 2014, 8: 1497-1508

18 Okada T, Miller MJ, Parker I, Krummel MF, Neighbors M, Hartley SB, O'Garra A, Cahalan MD, Cyster JG. Antigen-engaged B cells undergo chemotaxis toward the $\mathrm{T}$ zone and form motile conjugates with helper T cells. PLoS Biol, 2005, 3: e150

19 Qi H, Cannons JL, Klauschen F, Schwartzberg PL, Germain RN. SAP-controlled T-B cell interactions underlie germinal centre formation. Nature, 2008, 455: 764-769

20 Kerfoot SM, Yaari G, Patel JR, Johnson KL, Gonzalez DG, Kleinstein SH, Haberman AM. Germinal center B cell and T follicular helper cell development initiates in the interfollicular zone. Immunity, 2011, 34: 947-960

21 Kitano M, Moriyama S, Ando Y, Hikida M, Mori Y, Kurosaki T, Okada T. Bcl6 protein expression shapes pre-germinal center B cell dynamics and follicular helper $\mathrm{T}$ cell heterogeneity. Immunity, 2011, 34: 961-972

22 McHeyzer-Williams M, Okitsu S, Wang N, McHeyzer-Williams L. Molecular programming of B cell memory. Nat Rev Immunol, 2012, 12: $24-34$

23 Allen CD, Okada T, Cyster JG. Germinal-center organization and cellular dynamics. Immunity, 2007, 27: 190-202

24 Basso K, Dalla-Favera R. Germinal centres and B cell lymphomagenesis. Nat Rev Immunol, 2015, 15: 172-184

25 Cattoretti G, Chang CC, Cechova K, Zhang J, Ye BH, Falini B, Louie DC, Offit K, Chaganti RS, Dalla-Favera R. BCL-6 protein is expressed in germinal-center B cells. Blood, 1995, 86: 45-53

26 Fukuda T, Yoshida T, Okada S, Hatano M, Miki T, Ishibashi K, Okabe S, Koseki H, Hirosawa S, Taniguchi M, Miyasaka N, Tokuhisa T. Disruption of the BCL6 gene results in an impaired germinal center formation. J Exp Med, 1997, 186: 439-448

27 Phan RT, Dalla-Favera R. The BCL6 proto-oncogene suppresses p53 expression in germinal-centre B cells. Nature, 2004, 432: 635-639

28 Ranuncolo SM, Polo JM, Dierov J, Singer M, Kuo T, Greally J, Green R, Carroll M, Melnick A. Bcl-6 mediates the germinal center B cell phenotype and lymphomagenesis through transcriptional repression of the DNA-damage sensor ATR. Nat Immunol, 2007, 8: 705-714

29 Phan RT, Saito M, Basso K, Niu H, Dalla-Favera R. BCL6 interacts with the transcription factor Miz-1 to suppress the cyclin-dependent kinase inhibitor p21 and cell cycle arrest in germinal center B cells. Nat Immunol, 2005, 6: 1054-1060

30 Fujita N, Jaye DL, Geigerman C, Akyildiz A, Mooney MR, Boss JM, Wade PA. MTA3 and the Mi-2/NuRD complex regulate cell fate during B lymphocyte differentiation. Cell, 2004, 119: 75-86

31 Shaffer AL, Yu X, He Y, Boldrick J, Chan EP, Staudt LM. BCL-6 represses genes that function in lymphocyte differentiation, inflammation, and cell cycle control. Immunity, 2000, 13: 199-212

32 Allman D, Jain A, Dent A, Maile RR, Selvaggi T, Kehry MR, Staudt LM. BCL-6 expression during B-cell activation. Blood, 1996, 87: 5257-5268

33 Ci W, Polo JM, Cerchietti L, Shaknovich R, Wang L, Yang SN, Ye K, Farinha P, Horsman DE, Gascoyne RD, Elemento O, Melnick A. The BCL6 transcriptional program features repression of multiple oncogenes in primary B cells and is deregulated in DLBCL. Blood, 2009, 113: 5536-5548

34 Lo Coco F, Ye BH, Lista F, Corradini P, Offit K, Knowles DM, Chaganti RS, Dalla-Favera R. Rearrangements of the BCL6 gene in diffuse large cell non-Hodgkin's lymphoma. Blood, 1994, 83: $1757-1759$

35 Cattoretti G, Pasqualucci L, Ballon G, Tam W, Nandula SV, Shen Q, Mo T, Murty VV, Dalla-Favera R. Deregulated BCL6 expression recapitulates the pathogenesis of human diffuse large B cell lymphomas in mice. Cancer Cell, 2005, 7: 445-455

36 Huynh KD, Fischle W, Verdin E, Bardwell VJ. BCoR, a novel corepressor involved in BCL-6 repression. Genes Dev, 2000, 14: $1810-1823$

37 Ahmad KF, Melnick A, Lax S, Bouchard D, Liu J, Kiang CL, Mayer S, Takahashi S, Licht JD, Prive GG. Mechanism of SMRT corepressor recruitment by the BCL6 BTB domain. Mol Cell, 2003, 12: 1551-1564

38 Karagianni P, Wong J. Hdac3: taking the SMRT-N-CoRrect road to repression. Oncogene, 2007, 26: 5439-5449

39 Ghetu AF, Corcoran CM, Cerchietti L, Bardwell VJ, Melnick A, Privé GG. Structure of a BCOR corepressor peptide in complex with the BCL6 BTB domain dimer. Mol Cell, 2008, 29: 384-391

40 Gearhart MD, Corcoran CM, Wamstad JA, Bardwell VJ. Polycomb group and SCF ubiquitin ligases are found in a novel BCOR complex that is recruited to BCL6 targets. Mol Cell Biol, 2006, 26: 6880-6889

41 Hatzi K, Jiang Y, Huang C, Garrett-Bakelman F, Gearhart MD, Giannopoulou EG, Zumbo P, Kirouac K, Bhaskara S, Polo JM, Kormaksson M, MacKerell AD Jr, Xue F, Mason CE, Hiebert SW, Prive GG, Cerchietti L, Bardwell VJ, Elemento O, Melnick A. A hybrid mechanism of action for BCL6 in B cells defined by formation of functionally distinct complexes at enhancers and promoters. Cell Rep, 2013, 4: 578-588

42 Bereshchenko OR, Gu W, Dalla-Favera R. Acetylation inactivates the transcriptional repressor BCL6. Nat Genet, 2002, 32: 606-613

43 Mendez LM, Polo JM, Yu JJ, Krupski M, Ding BB, Melnick A, Ye $\mathrm{BH}$. CtBP is an essential corepressor for BCL6 autoregulation. Mol 
Cell Biol, 2008, 28: 2175-2186

44 Basso K, Saito M, Sumazin P, Margolin AA, Wang K, Lim WK, Kitagawa Y, Schneider C, Alvarez MJ, Califano A, Dalla-Favera R. Integrated biochemical and computational approach identifies BCL6 direct target genes controlling multiple pathways in normal germinal center B cells. Blood, 2010, 115: 975-984

45 Huang C, Geng H, Boss I, Wang L, Melnick A. Cooperative transcriptional repression by BCL6 and BACH2 in germinal center B-cell differentiation. Blood, 2014, 123: 1012-1020

46 Reljic R, Wagner SD, Peakman LJ, Fearon DT. Suppression of signal transducer and activator of transcription 3-dependent B lymphocyte terminal differentiation by BCL-6. J Exp Med, 2000, 192: 1841-1848

47 Cerchietti LC, Polo JM, Da Silva GF, Farinha P, Shaknovich R, Gascoyne RD, Dowdy SF, Melnick A. Sequential transcription factor targeting for diffuse large B-cell lymphomas. Cancer Res, 2008, 68:
3361-3369

48 Parekh S, Polo JM, Shaknovich R, Juszczynski P, Lev P, Ranuncolo SM, Yin Y, Klein U, Cattoretti G, Dalla Favera R, Shipp MA, Melnick A. BCL6 programs lymphoma cells for survival and differentiation through distinct biochemical mechanisms. Blood, 2007, 110: 2067-2074

49 Cerchietti LC, Yang SN, Shaknovich R, Hatzi K, Polo JM, Chadburn A, Dowdy SF, Melnick A. A peptomimetic inhibitor of BCL6 with potent antilymphoma effects in vitro and in vivo. Blood, 2009, 113: 3397-3405

50 Green JA, Suzuki K, Cho B, Willison LD, Palmer D, Allen CD, Schmidt TH, Xu Y, Proia RL, Coughlin SR, Cyster JG. The sphingosine 1-phosphate receptor $\mathrm{S}_{2} \mathrm{P}_{2}$ maintains the homeostasis of germinal center B cells and promotes niche confinement. Nat Immunol, 2011, 12: 672-680

Open Access This article is distributed under the terms of the Creative Commons Attribution License which permits any use, distribution, and reproduction in any medium, provided the original author(s) and source are credited. 